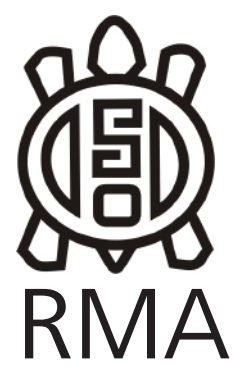

Dossier

\title{
Dietas promediadas: explorando el registro zooarqueológico supra- regional en Tierra del Fuego
}

\author{
Fernando Santiago* y Martín Vázquez**
}

* CADIC-CONICET. Ushuaia. E-mail: ersant2@gmail.com ** Museo del Fin del Mundo. Ushuaia. E-mail: vazquez_martin@speedy.com.ar

\begin{abstract}
Resumen
En este trabajo se compila información zooarqueológica proveniente de 97 contextos correspondientes a 75 sitios arqueológicos emplazados en distintas regiones del archipiélago fueguino, con cronologías que abarcan desde el Holoceno temprano hasta el contacto con la sociedad occidental. Por medio de la utilización de SIG (Sistemas de información geográfica) e índices de abundancia económica se suman los NISP de conjuntos de un espacio determinado, lapso o cultura y se calcula un nuevo índice de abundancia. De esta manera se generan valores promediados de consumo de fauna para una región o para un momento en particular del pasado. En este trabajo se utiliza una escala supraregional para comprender de modo no convencional como se estructuran los distintos conjuntos zooarqueológicos en su contexto natural y cultural.
\end{abstract}

Palabras clave: Zooarqueología; Tierra del Fuego; Análisis supraregional; Espacio promediado.

Averaged diets: exploring the supra-regional zooarchaeological record in Tierra del Fuego

\begin{abstract}
This paper compiles information from 97 zooarchaeological contexts from 75 archaeological sites placed in different regions of Tierra del Fuego archipelago, with chronologies ranging from the Early Holocene to the first contact with Western society. By means of GIS (Geographic Information Systems) analysis and abundance indexes based on NISP values, the abundance of different taxa through space is assessed. This generates average values of consumption of wildlife in a region or at a particular time in the past. Whereas most zooarchaeology analyses are site-specific, this paper uses a supra-regional scale to understand the zooarchaeological structure in its natural and cultural context.
\end{abstract}

Key Words: Zooarchaeology; Tierra del Fuego; supraregional analysis; Space averaging.

La arqueología de Tierra del Fuego está marcada por un fuerte contraste entre dos tradiciones cazadorasrecolectoras: los cazadores pedestres que ocuparon el norte y centro de la Isla Grande, basando su modo de vida en la explotación del guanaco complementado con recursos costeros; y los canoeros con un modo de vida basado sobre la cacería de pinnípedos y la explotación de otros recursos litorales. Hacia tiempos recientes estas tradiciones desembocaron en cuatro etnias bien conocidas en la literatura etnográfica. Por una parte, los Selk'nam, cazadores pedestres ocupando el norte y centro del territorio fueguino y los Haush, en península Mitre, pero con una mayor dependencia de los recursos litorales. Por otra parte, emplazados en la región de los canales e islas al sur y oeste del archipiélago Magallánico Fueguino, los Yámanas y Alakalufes, ambos canoeros con una fuerte dependencia de los recursos litorales. Durante las últimas décadas, la ampliación de los estudios zooarqueológicos, en conjunción con otras líneas de evidencia, como por ejemplo los análisis isotópicos llaman la atención sobre la rigidez de estos modelos previos (Barberena 2004, Zangrando 2009, Santiago 2010, Borrero 2011, Vázquez et al. 2011).

En este sentido, en esta revisión y análisis de la información publicada se aplica un enfoque exploratorio de escala supraregional. Se compila información zooarqueológica de 97 contextos correspondientes a 75 sitios emplazados en distintas regiones del archipiélago fueguino y de cronologías variables.

El objetivo es realizar un análisis a escala supraregional del consumo prehistórico de fauna; por medio de la utilización de índices de abundancia económica (Lyman 2003) y sistemas de información geográfica. 


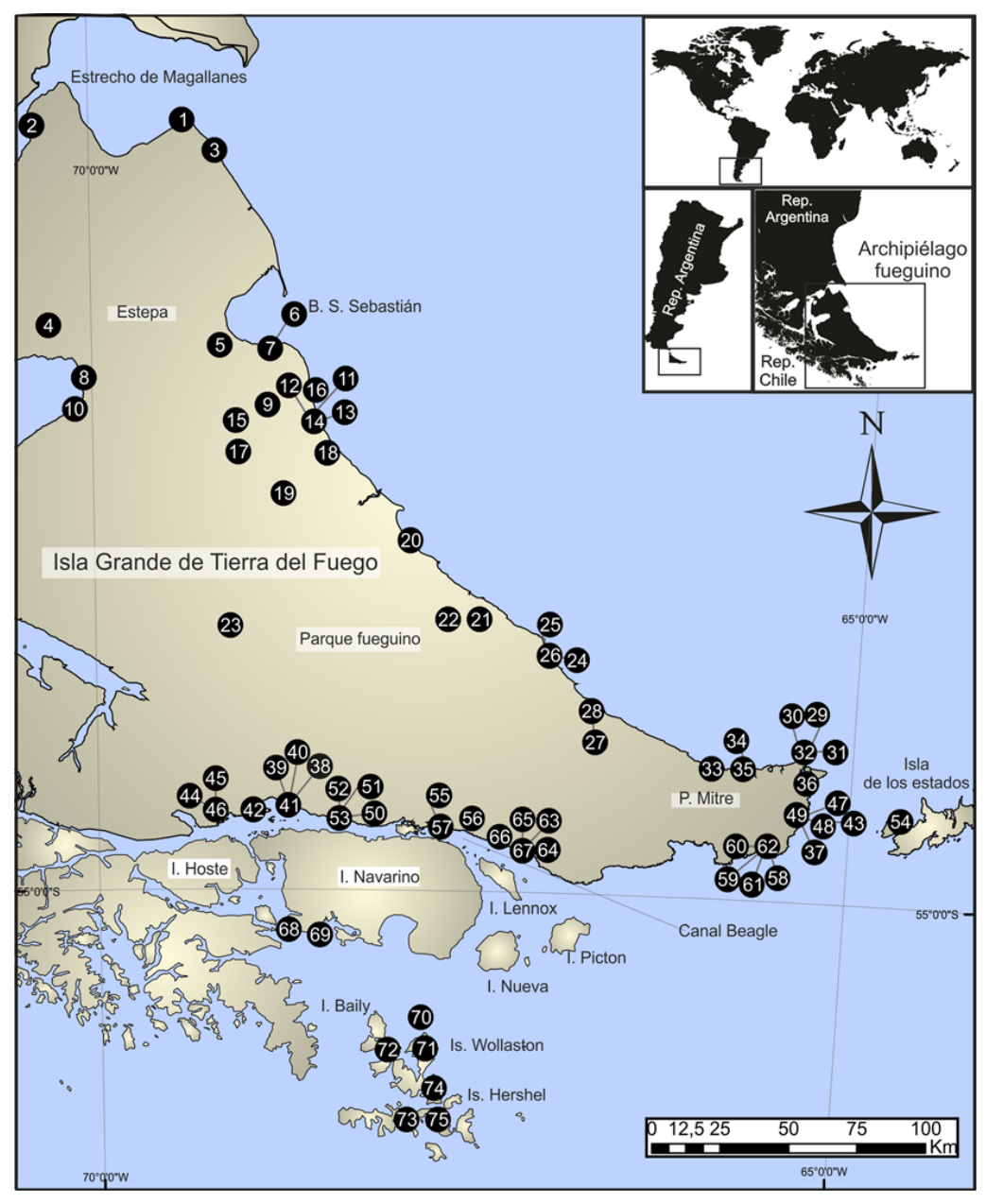

Figura 1: Mapa del archipiélago fueguino, los círculos numerados indican las ubicaciones de los sitios arqueológicos analizados (los números se corresponden con la segunda columna de la tabla 1).

Figure 1: Map of Tierra del Fuego, the numbered circles indicate the locations of archaeological sites analyzed (numbers correspond to the second column of Table 1).

contextos, son muy escasos y carecen de estudios detallados.

Hacia el Holoceno Medio, completado el proceso de insularidad y por ende con la existencia de una barrera biogeografía de la magnitud del Estrecho de Magallanes, la señal arqueológica se hace paulatinamente más fuerte y diversa. Este proceso coincide con una serie de trasformaciones ambientales: la expansión del bosque de Nothofagus (Ponce et al. 2011), una tendencia hacia un clima relativamente más frío en toda Patagonia (Heusser 2003) y una elevación de los niveles de costa (Bujalesky 2007). En toda la Patagonia las trayectorias evolutivas locales y regionales muestran un proceso de marcada diferenciación. Según el modelo de poblamiento planteado por Borrero (1989), esta diferenciación de estrategias adaptativas, ejemplificadas con

\section{La explotación de vertebrados en Tierra del Fuego}

Para el límite Pleistoceno-Holoceno (ca. 12.000-9.000 AP) en el extremo sur de la Patagonia hay un relativo consenso en que la subsistencia estuvo centrada en la explotación del guanaco y el aprovechamiento oportunista de especies extinguidas (para una revisión ver Borrero 2005). En territorio fueguino los análisis faunísticos y tafonómicos llevados a cabo en las capas inferiores de Tres Arroyos (ca. 11.500 AP) se ajustan a este patrón y la bajas frecuencias de huellas de origen antrópico indican que el procesamiento de las presas no habría sido intensivo (Borrero 2003, Massone 2010). Para los primeros milenios del Holoceno la señal arqueológica en la Isla Grande es sumamente débil o prácticamente inexistente (LamingEmperaire et al. 1972, Massone 2004, Massone 2010). Los hallazgos fechados en este intervalo se reducen a un solo sitio: Marazzi 1, cuyos niveles inferiores fueron fechados en 9.590 años AP (Laming-Emperaire et al. 1972); aunque la naturaleza de la asociación entre el fechado y las evidencias de ocupación humanas han sido discutidas (Morello et al. 1999).

Los contextos siguientes se registran en la margen norte del canal Beagle: Primer Componente de Túnel I con fechas entre 6980 y 6680 (Orquera y Piana 1988, 1999) y Capa S de Imiwaia fechada en $7842 \pm 53$ (Zangrando 2010). En cuanto a los restos óseos provenientes de estos el denominado "Casapedrense" en Patagonia Continental y por la aparición de las adaptaciones marítimas en el Archipiélago Fueguino, coincidiría con la denominada Fase de Colonización. Paralelamente, en diversos conjuntos faunísticos de la Patagonia fechados entre los 8500 y 5000 años AP fue señalada una reducción de especies cazadas con respecto al Pleistoceno tardío/ Holoceno temprano 13000/8500 años AP. (Miotti y Salemme 1999). Este cambio estaría relacionado con la reducción numérica de especies, consecuencia de las extinciones de fines del Pleistoceno, y con un giro en las estrategias de subsistencia hacia la caza del guanaco. Por su parte, en Tierra del Fuego, una de las características de este período es la incorporación a las arqueofaunas de los pinnípedos y otros recursos costeros entre los 7.000/5.000 años AP, en primer término relacionado con la aparición de contextos canoeros en el archipiélago; y en segundo término debido a las claras evidencias de aprovechamiento del litoral atlántico en el norte de la Isla Grande (Orquera y Piana 1999, 2009, Salemme et al. 2007, Santiago 2010, Muñoz 2004).

Si bien más numerosos y con mayor disponibilidad de información zooarqueológica, que los registros previos a los 6.000 años, los contextos del Holoceno Medio son aún escasos. Entre estos pueden destacarse los contextos canoeros tempranos de la región del canal Beagle: $2^{\text {do }}$ componente de Túnel, Componente Antiguo 
de Lancha Pakewaia, Capa K y M de Imiwaia y Capa F de Mischiúen (Orquera y Piana 1999, Piana et al. 2004); otros sitios costeros emplazados en la costa Atlántica: La Arcillosa 2 y Rio Chico 1 (Salemme et al. 2007, Santiago 2010) etc., en la pacífica como Marazzi 1 y el contexto temprano del Sitio 11 de Bahía Valentín (Vázquez et al. 2011), todos estos fechados en el lapso $6.400-4.000$ AP. Posteriormente, entre los 4.000 y los 2.000 años AP las cantidades de contextos registrados se reducen drásticamente.

La mayor parte de los yacimientos estudiados en Tierra del Fuego corresponden a los últimos dos milenios, dentro de estas fechas se encuentra la mayor abundancia de datos faunísticos. En consecuencia la gran mayoría de los modelos propuestos para las distintas regiones del archipiélago fueguino han sido construidos a partir de esta evidencia. Salvo en la región del Beagle, no existen análisis de escala regional que evalúen la existencia de cambios y tendencias temporales en el aprovechamiento de los recursos faunísticos (Zangrando 2010, Tivoli 2010).

Para el norte y centro de la Isla Grande, Borrero (1985) propone un modelo de alta movilidad residencial y baja intensidad ocupacional, sin marcados patrones estacionales en la explotación de los recursos. En este modelo se sostiene que guanacos y pinnípedos habrían sido los recursos críticos del sistema de subsistencia. Si bien el rol principal del recurso guanaco es incuestionable, ya que sus restos son dominantes en los conjuntos del interior, los asentamientos costeros se caracterizan por el énfasis en la explotación de recursos litorales, entre los que sobresalen los pinnípedos. Los sitios del interior son generalmente pequeños y se distribuyen en forma relativamente homogénea en el espacio. Por el contrario, en los espacios litorales se registran sitios de mayores dimensiones y una cierta redundancia ocupacional en determinados sectores, lo que lleva al autor a sostener que las ocupaciones costeras y sus recursos jugaron un rol importante en la economía prehistórica de estos grupos humanos (Borrero 1985: 303-305). No obstante, investigaciones posteriores indican que esta tendencia en la dominancia de pinnípedos en conjuntos del litoral atlántico no es tan constante: casos como Espíritu Santo 1 y San Genaro 1 presentan claras dominancias de restos de guanacos sobre los de pinnípedos (Horwitz 2004). Estas nuevas evidencias ponen de manifiesto un nivel mayor de complejidad en los patrones de subsistencia que los propuestos inicialmente. Esto también se ve reflejado en los valores isotópicos sobre restos humanos (Guichón et al. 2001, Yesner et al. 2003, Barberena 2004, Panarello et al. 2006, Santiago et al. 2011).

En el planteo original (Borrero 1985), las cacerías de guanacos habrían sido de escala reducida, principalmente por intercepción, aprovechando senderos, terrenos quebrados o claros del bosque. Este modelo sostenía la ausencia de cacerías masivas, que implicaran algún tipo de organización logística y la matanza de muchos individuos en un mismo episodio (Borrero 1985, 1990; Muñoz 2004). No obstante, la evidencia proveniente del sitio Las Vueltas (Santiago 2010) sugiere la existencia de eventos de caza masiva en lugares donde estas actividades se viesen favorecidas por la topografía. Esta evidencia aporta un grado más de variabilidad a la alternativa de cacerías en pequeña escala, señalando que en determinadas configuraciones situacionales, los grupos humanos se habrían organizado para la ejecución de un plan de cacería más complejo, lo que implicaría otras condiciones de transporte, reparto, etc.

Los guanacos no habrían sido transportados en forma completa desde su lugar de obtención y faenamiento primario a los sitios de consumo, sino mediando una cierta selección de partes (Borrero 1985, 1990). Por el contrario los pinnípedos habrían sido transportados en forma completa (Muñoz 2004).

El sudeste de la Isla Grande de Tierra del Fuego, conocido bajo el nombre de península Mitre, presenta un panorama más complejo que el sector norte de la isla. En primer lugar las características del paisaje permiten establecer la diferenciación en dos espacios bien definidos. Por un lado, la costa atlántica fácilmente transitable por tierra a lo largo de sus extensas playas, presenta grandes dificultades a la navegabilidad, agravadas por un régimen de mareas de gran amplitud. Por el contrario, la costa meridional, recortada y escarpada dificulta el tránsito terrestre, pero la menor aptitud de mareas, presencia de bosques y su continuidad con la región de los canales interiores genera una mayor expectativa de vinculación por vía marítima con esta última región.

A partir de la evidencia recuperada en la costa norte de península Mitre, ubicada cronológicamente dentro de los últimos 1.500 años, se generó un modelo arqueológico de escala regional que aborda distintos aspectos del registro (Lanata 1995, Muñoz y Belardi 2011). Sobre la base de la ausencia de evidencia de medios de navegación y de instrumental de obtención especializado, se postula que la accesibilidad a los pinnípedos habría sido desde tierra. Lanata y Winograd estiman que para momentos anteriores a su explotación industrial, en Tierra del Fuego el 50\% de la población de pinnípedos debe haber estado emplazada entre la desembocadura del río Policarpo y bahía Aguirre $y$, que si bien con ciertas fluctuaciones, este recurso habría estado disponible en los apostaderos durante todo el año (Lanata y Winograd 1988, Lanata 1995, 1996). Las variaciones estacionales estarían vinculadas a su ciclo reproductivo: en verano los grupos de pinnípedos con sus crías tendrían una dependencia más marcada a los apostaderos y por ende un comportamiento más pautado y predecible y hacia otoño-invierno disminuiría su vinculación con la costa y su comportamiento sería menos predecible Independientemente de la técnica o el armamento utilizado, se prevén dos situaciones de obtención diferenciadas: encuentros aleatorios de cazadores-presas y la búsqueda intencional en forma de 
incursiones de cacería sobre apostaderos específicos, en las cuales se obtendrían diferentes clases de sexo y edad (Lanata 1996, Lanata y Winograd 1988).

En esta región los guanacos no presentan un patrón estacional definido y las estrategias de obtención estarían más vinculadas a las pautas etológicas de este recurso. Aquí los grupos familiares, de comportamiento más predecible, serían explotados en forma más intensiva hacia otoño-invierno y los grupos de machos hacia la primavera-verano. En cuanto a los modos de obtención de estas presas, las estrategias serian de la misma clase que las propuestas por Borrero para el norte de la Isla; es decir: cacerías por intercepción de uno o unos pocos individuos a la vez.

En síntesis, los dos recursos críticos para la subsistencia, pinnípedos y camélidos, serían relativamente predecibles y su disponibilidad no presentaría mayores discontinuidades estacionales. Los pinnípedos sufrirían un incremento en la explotación hacia el verano y principios de otoño y los guanacos estarían disponibles durante todo el año. La localización de los pinnípedos, acotada a espacios definidos de la costa, en comparación con una dispersión mayor de los grupos de guanacos, generaría expectativas de menor inversión de esfuerzos en la localización, persecución y obtención, así como de menor intensidad de trozamiento primario y trasporte de los pinnípedos frente a los guanacos (Muñoz 2002, 2004).

Los estudios realizados hasta el momento en la costa suroriental de península Mitre indican condiciones variables tanto en relación a los emplazamientos como a las proporciones taxonómicas en que se componen las arqueofaunas, aparentando una cierta tendencia a variar en función de las características fisiográficas y la oferta local de recursos (Zangrando et al. 2009, Vázquez et al. 2011).

La característica sobresaliente de los conjuntos faunísticos del canal Beagle es, sin lugar a dudas, su diversidad taxonómica (Orquera y Piana 1999; Piana et al. 2009). La subsistencia de los canoeros que habitaron esta región ha incluido una gran variedad de invertebrados (moluscos, crustáceos y equinodermos), así como de vertebrados marinos y terrestres (pinnípedos, camélidos, aves, peces y cetáceos). La explotación de tal variedad de recursos implicó el desarrollo de una importante diversidad de estrategias de obtención, transporte y procesamiento según fuese la presa: cacerías en agua y tierra, recolección, carroñeo, pesca. En consecuencia, también el desarrollo de una multiplicidad de medios tecnológicos destinados a estos fines: arpones de punta separable y fija, armas arrojadizas, trampas, contenedores, redes, etc. (Orquera y Piana 1999).

Se postula que la subsistencia de estos grupos a lo largo de sus 6 milenios de historia estuvo basada principalmente sobre la explotación de los pinnípedos y que los demás taxa habrían funcionado a manera de complemento (Orquera y Piana 1999; Schiavini 1993; Piana et al. 2009). El desarrollo de medios tecnológicos como arpones de punta separable, presentes a lo largo de toda la secuencia, constituye un indicador confiable de la obtención de estas presas en medio acuático. Los perfiles de mortalidad de las presas cazadas en la mayoría de los casos reflejan una predominancia de machos cazados en los meses invernales, lo que se opone a lo esperable si las cacerías se efectuaran en roquerías o colonias reproductivas y la representación de todas las partes anatómicas, sugiere el transporte de las presas enteras al sitio (Orquera y Piana 1999).

Los restos de aves son ubicuos en los conjuntos de la región, y aunque su tamaño corporal implica bajos niveles de retorno calórico por presa, en determinadas circunstancias los individuos se encuentran agrupados -colonias reproductivas, presencia de cardúmenes de peces que pueden atraer aves ictiófagas, etc.- lo que genera condiciones favorables para obtención de muchos individuos. Generalmente los taxa mejor representados son los cormoranes (Phalacrocórax sp.) y los pingüinos. De estos últimos fundamentalmente se han registrado dos especies: Spheniscus magellanicus y Eudyptes crestatus, siendo el primero de ellos, por mucho, el mejor representado. En los conjuntos también se registran otras taxa en proporciones menores: proceláridos, Diomedeidae y anátidos (Tivoli 2011).

En esta región la distribución del guanaco está restringida a la costa sur de la Isla Grande de Tierra del Fuego e Isla Navarino y sus restos son relativamente comunes en muchos contextos excavados, no obstante su explotación por parte de los canoeros tiene que haber estado restringida por su distribución. Orquera y colaboradores (2008) sostienen que la las proporciones de guanacos en las arqueofaunas aumentan en configuraciones topográficas favorables para su encuentro, por ejemplo hacia la porción este del canal (Orquera et al. 2008: 277). Estas presas en la mayoría de los casos debieron haber sido cazadas dentro del bosque, lo que implicaría el faenamiento en localizaciones distantes a los sitios de habitación y el consecuente transporte de ciertas partes para su consumo (Orquera y Piana 1996, 1999).

Las aguas del canal Beagle albergan cantidad de peces; si bien el rendimiento individual es bajo, en temporada estival ingresan a las aguas interiores grandes cardúmenes de especies pelágicas como las merluzas (Merlucciius hubbsi) y sardinas (Sprattus fueguensis). Incluso se registran en forma recurrente varamientos de estos cardúmenes, lo que debe haber significado una oferta masiva de alimento a muy bajos costos de obtención (Zangrando 2009). Por otro lado también existen otras especies de peces presentes todo el año en aguas someras y accesibles desde la costa o desde las canoas. 
El aprovechamiento de cetáceos estuvo condicionado a la existencia de sus varamientos; estos animales brindaban una importante cantidad de alimento con alto rendimiento calórico por individuo, además del valor de sus huesos para utilizarlos como materia prima. Si bien en relativamente bajas frecuencias, los restos óseos de cetáceos se registraron prácticamente en todos los yacimientos de la región del Beagle.

\section{Materiales y métodos}

La escala del análisis utilizada en este trabajo puede considerarse supraregional, ya que el ámbito geográfico analizado abarca la totalidad del Archipiélago Fueguino -Isla Grande, Navarino, Isla de los Estados, islas Baily y Wollaston- (Fig. 1). En la Tabla 1 se enumeran los contextos incluidos en este análisis, los NISP de cada uno, los índices de abundancia calculados (IA), las fuentes bibliográficas de referencia y el lapso que representan y en la figura 1 se puede observar la ubicación de los sitios incluidos en este trabajo.

Para este análisis se recopilaron datos provenientes de 75 sitios, que incluyen 97 conjuntos arqueofaunísticos diferenciados. Para la definición de estos contextos se tuvieron en cuenta las propuestas de los diferentes autores basados sobre los datos estratigráficos y fechados radiocarbónicos disponibles. Estos conjuntos incluyen un número total de 257994 restos faunísticos de vertebrados, excluyendo para los fines de este análisis los restos óseos no identificados y los de invertebrados.

De estos 75 sitios, 66 se encuentran emplazados en la Isla Grande de Tierra del Fuego (88 contextos), 2 en la isla de Navarino (2 contextos), 1 en la isla de los Estados (1 contexto) y 6 en el archipiélago del Cabo de Hornos (6 contextos). El lapso abarcado por los conjuntos estudiados abarca desde 8000 años AP, hasta el contacto con el mundo occidental.

La información faunística disponible para Tierra del Fuego es abundante, sin embargo el nivel de análisis en que se han tratado los diferentes conjuntos, así como la información disponible son muy heterogéneos, lo que dificulta efectuar comparaciones o análisis integradores. En algunos casos solo se cuenta con menciones superficiales y cualitativas sobre la presencia de determinados taxones y otros conjuntos cuentan con detallados informes sobre aspectos taxonómicos, anatómicos y tafonómicos. Asimismo en muchos casos en los cuales se presentan estimaciones basadas sobre determinaciones anatómicas de los especímenes (por ejemplo NMI o NME), no siempre se especifican los criterios sobre los cuales se basaron estos cálculos. Las potenciales variaciones de estas estimaciones según se incluyan o no criterios como: clases de edad, tamaño o dimorfismo sexual podrían tornar en incomparables las diferentes situaciones, más aún en taxones con un gran espectro de variabilidad como los pinnípedos.

En este sentido, en función del enfoque supraregional de este trabajo y de la necesidad de incluir el mayor número posible de conjuntos, nos hemos visto obligados a realizar este análisis sobre la base del NISP. No obstante, conscientes de las dificultades de derivar directamente de estos valores patrones de subsistencia, consideramos que es la única medida de abundancia taxonómica disponible en la gran mayoría de los análisis y cuyo modo de cálculo es unívoco, por lo tanto la más adecuada para estos fines (Grayson 1984; Lyman 2003, 2008).

Existe también una serie de factores y aspectos culturales, tafonómicos y metodológicos que deben haber influenciado las representación de los taxones en los conjuntos fueguinos. Para el caso de camélidos y pinnípedos, las pautas de transporte diferencial de partes propuestas para los guanacos y, por el contrario, la tendencia al trasporte completo de los pinnípedos, tenderían a la sobrerrepresentación de las frecuencias de los segundos. Por el contario, las pautas de fractura de huesos largos de guanacos para el consumo de medula ósea, frente a su ausencia en pinnípedos, tenderían a aumentar las frecuencias del NISP de camélidos. Otro aspecto a tener en cuenta es la conservación diferencial de elementos pequeños o de baja densidad como podrían ser el caso de los restos de peces, estos últimos especialmente sensibles a las técnicas de recuperación que podrían disminuir o sobre-representar sus frecuencias significativamente. Para el caso de los cetáceos, ya sea el ingreso a los sitios de carne o grasa sin hueso o bien la utilización de los huesos como materia prima, pueden dificultar la interpretación de las situaciones de consumo.

Del análisis de la información zooarqueológica disponible se pudo constatar la presencia en frecuencias variable de ocho categorías taxonómicas predominantes para los conjuntos arqueofaunísticos fueguinos: pinnípedos, guanacos, aves, cetáceos, peces, roedores, cánidos y mustélidos.

Para efectuar los análisis se utilizó como herramienta metodológica el Índice de Abundancia (IA) (Lyman 2003), éste se calculó para cada una de las categorías y conjuntos, a fin de monitorear los cambios en la abundancia de presas a través del tiempo y del espacio. La fórmula empleada para el cálculo del índice de Abundancia es la siguiente:

\section{$\sum$ NISPguanacos}

(โNISPguanaco $+\sum$ NISPaves $+\sum$ NISPpinn $+\sum$ NISPpeces $+\sum$ NISPcetáceos $+\sum$ NISProedores $+\sum$ NISPcánidos $+\sum$ NISPmustélidos $)$

Los valores del índice son resultados normalizados que oscilan entre 0 y 1, valores mayores e iguales a 0,5 indican predominancia del recurso y valores menores a 0,5 y cercanos a 0 indican ausencia del recurso en cuestión (Lyman 2003, 2008). 


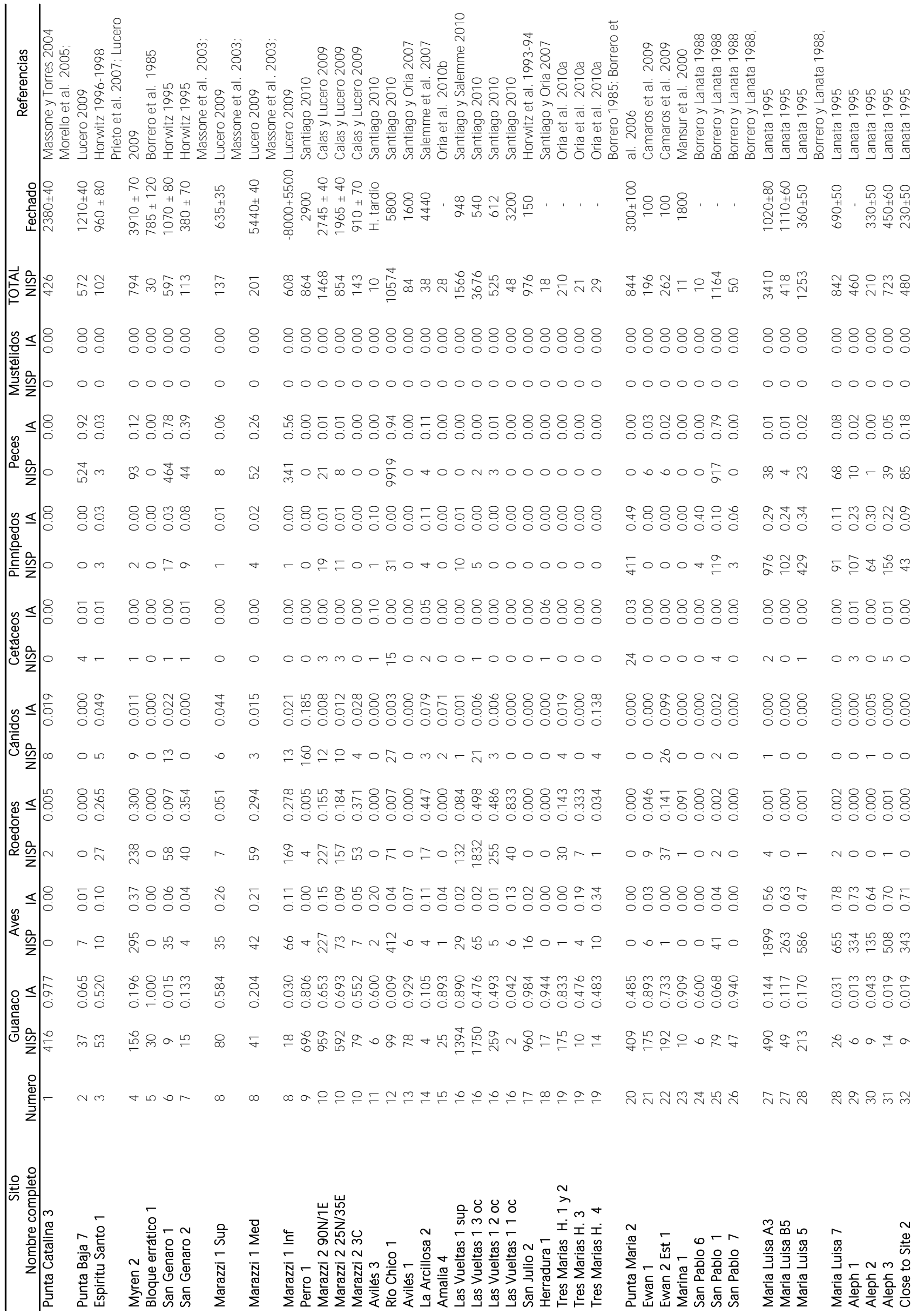




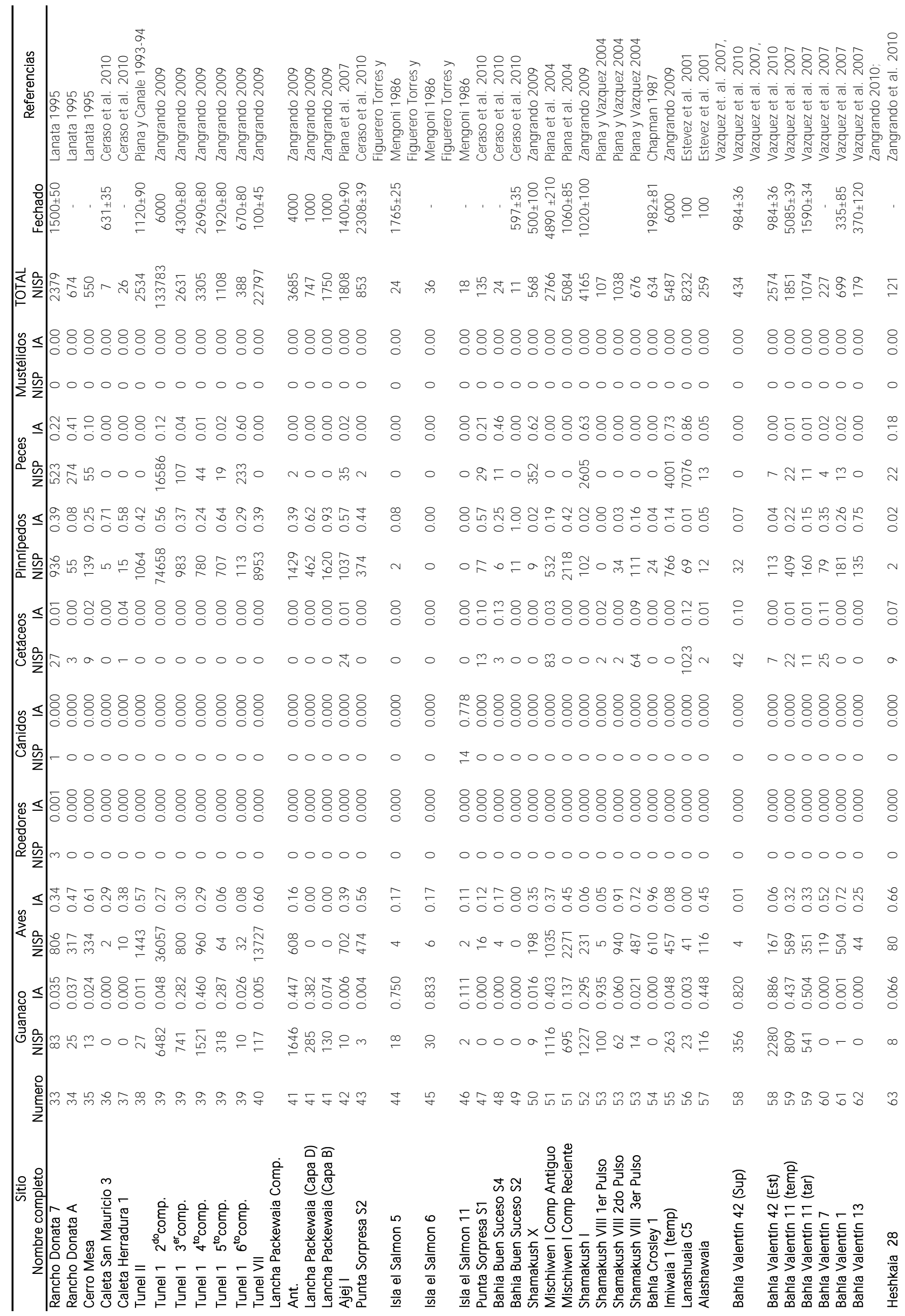




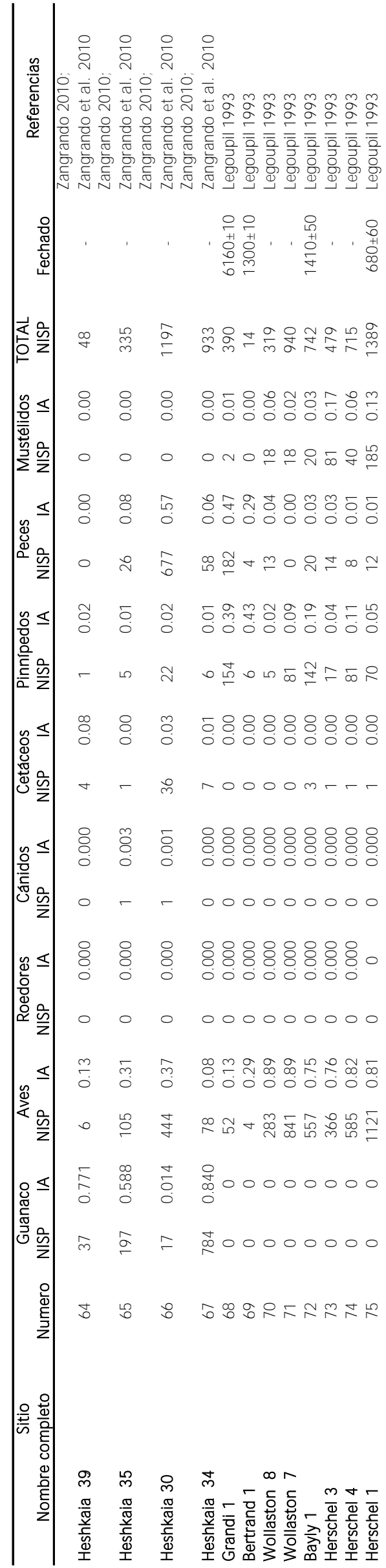

Tabla 1: Sumario de sitios arqueológicos, NISP por taxones analizados, índices de abundancia calculados, fechados radiocarbónicos y referencias bibliográficas.

Table 1: Summary of archaeological sites, NISP by analyzed taxa, abundance index calculated, radiocarbon dates and references.

\section{Resultados}

Si tenemos en cuenta solamente la presencia o ausencia de determinados recursos en los sitios considerados podemos constatar que en el 89,7\% de los 97 conjuntos arqueofaunísticos se identificaron restos óseos de aves $(\mathrm{N}=96)$, en orden decreciente se identificaron pinnípedos $(\mathrm{N}=88)$, guanacos $(N=83)$, peces $(N=66)$, cetáceos $(N=51)$, roedores $(N=41)$, cánidos $(\mathrm{N}=34)$ y mustélidos $(\mathrm{N}=18)$ (Fig. 1). Las tres primeras categorías podrían ser consideradas como la base de la economía fueguina, con distinta participación en la misma según en qué sector del archipiélago se encuentra el sitio arqueológico.

En cuanto a la representación global de especímenes para toda la muestra analizada, encontramos que las categorías mejor representadas son pinnípedos ( $\mathrm{N}=101.527)$, aves $(\mathrm{N}=75196)$, peces $(\mathrm{N}=45743)$ y guanacos $(\mathrm{N}=29824)$; seguidos en mucha menor frecuencia por roedores $(\mathrm{N}=3486)$, cetáceos ( $\mathrm{N}=1499)$, mustélidos $(\mathrm{N}=364)$ y cánidos $(\mathrm{N}=355)$.

Los pinnípedos, pese a su marcada preponderancia en el cálculo global, tienden a presentar valores bajos de NISP así como de IA, a excepción de la región del Beagle donde en general están bien representados o incluso son dominantes en varios conjuntos (Fig. 2c). Es necesario tener en consideración que los restos provenientes de la región del canal Beagle comprenden el $94 \%$ del total de los restos de pinnípedos contabilizados para todo Tierra del Fuego. Más aún, el valor de NISP que alcanza el $2^{\text {do }}$ Componente de Túnel I ( $\mathrm{N}=74.658$ ) por sí solo representa el 73\% (tabla 1). Por el contrario, las frecuencias de este taxón para el norte y centro de la Isla Grande son bajas (únicamente en el sitio PM2 se observa que el índice alcanza valores de 0,5), aumentando considerablemente hacia península Mitre, aunque sin alcanzar los altos valores de abundancia del Beagle.

Las aves aparecen en proporciones más homogéneas que los pinnípedos, aunque hacia las regiones del canal Beagle y península Mitre también se incrementa marcadamente su abundancia, en comparación con los conjuntos provenientes del norte y centro de la isla (Fig.2a). En los conjuntos del norte, salvo pocas excepciones, los valores de IA de aves no superan el 0.30 y si bien en general los conjuntos son más pequeños que los del Beagle la presentación absoluta de las aves también es baja. Por el contrario en península Mitre, ya sea la margen norte o la sur, así como también en el Beagle e islas Wollaston, numerosos conjuntos presentan una marcada dominancia de restos de aves y en más de una veintena de conjuntos el IA de aves supera el 0.5. Tomados en conjunto los restos de aves registrados en el canal Beagle, península Mitre e islas Wollaston

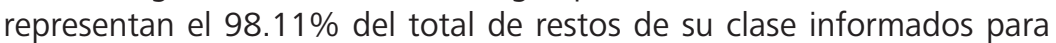
todo el archipiélago.

El guanaco es el taxón mas homogéneamente representado, a excepción claro está de su ausencia total en aquellos sectores del archipiélago donde no existen poblaciones de esta especie (Islas Wollaston, Bayly, Herschel e Isla de los Estados). Llama también la atención su ausencia en los dos contextos de la isla Navarino (Fig. 2b). Si bien una vez más los conjuntos de la región del Beagle superan, en valores absolutos, al resto de las regiones, 

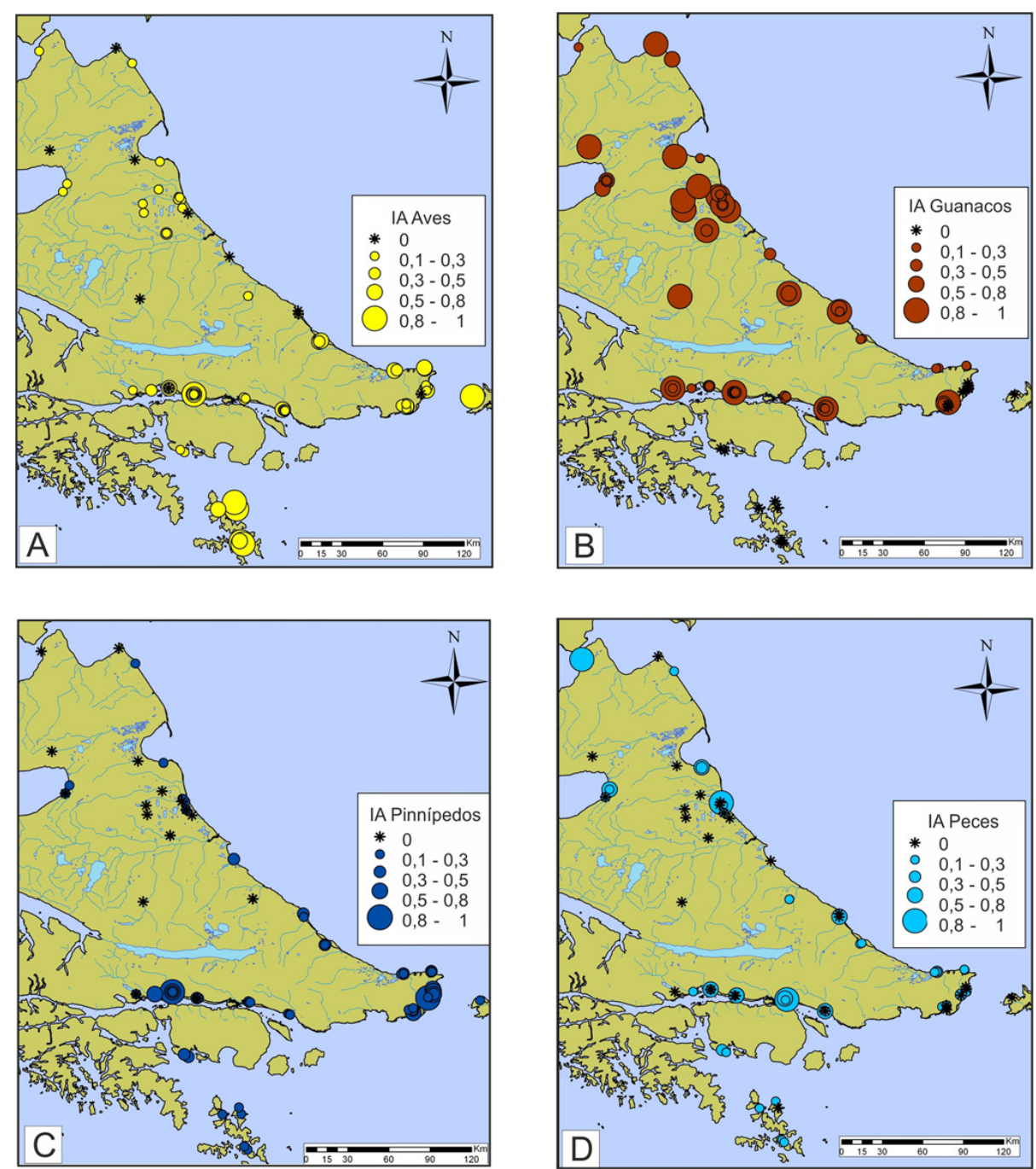

Figura 2: Mapas de distribución de A) índice de abundancia de aves. B) índice de abundancia de guanaco, C) índice de abundancia de pinnípedo, D) índice de abundancia de peces.

Figure 2: Distribution maps of A) bird index. B) guanaco index, C) Pinnipedia index, D) fish index.

de recuperación de esta clase de elementos (Zangrando 2009). No obstante, en todas las regiones existen casos donde los valores de IA de este recurso alcanzan cifras significativas, poniendo de manifiesto la importancia de su aporte a la subsistencia de los cazadores recolectores fueguinos (Fig. 2d). Este recurso se presenta en algunos sitios puntuales con altas cantidades de restos (e.g. PB7, RC1, Im I), la presencia del mismo es más discontinua, pero es el cuarto recurso en importancia si tenemos en cuenta su NISP total.

Los cetáceos muestran valores bajos para su índice en todos los contextos, y se presentan pocos especímenes por sitio, una excepción es el sitio Lanashuaia en donde se

en este caso sumados alcanzan un 51,3\% sobre el total; aquí los valores más altos de IA están representados en los sitios del Norte y centro de la Isla, donde en la mayoría de los casos superan el 0.5. En el canal Beagle presenta frecuencias variables, con algunos sitios con valores altos, como las localidades Isla el Salmon, Mischiúen/ Shamakush y Heshkaia, así como ciertos conjuntos de Túnel y Lancha Pakewaia.

En cuanto a la representación de esta especie, península Mitre presenta una clara diferenciación entre su costa norte y la sur. Para el caso de la primera, donde se puede verificar una clara dominancia de aves, las frecuencias relativas de restos de guanaco son muy escasas, para la costa sur, específicamente los sitios 11 y 42 de bahía Valentín, los restos de guanaco son abundantes y hasta dominantes.

El caso de los restos de peces posiblemente sea el que mayores sesgos de orden metodológico presenta, siendo difícil de aceptar que en algunos sitios costeros no se reporte ningún hallazgo de esta clase. En varias ocasiones se ha señalado la influencia que tienen los métodos de excavación, muestreo, tamizado, etc., sobre el potencial hallaron más de un millar de restos de cetáceos (Fig. 3a).

Para el caso de los taxones restantes cabe destacar algunos aspectos puntuales. Dos de ellos se presentan en espacios muy acotados. Es el caso de los roedores solo presentes en contextos del norte y centro de la Isla Grande (Fig. 3b), aunque sin dudas muchos de estos restos correspondan a especímenes incorporados a los contextos por causas naturales.

Los cánidos representados principalmente por dos especies se encuentran solamente presentes en bajas proporciones en los sitios de la estepa fueguina y excepcionalmente en el canal Beagle (Fig. 3c).

El otro caso es el de los mustélidos, solo documentados en sitios de las islas Wollaston y un caso al sur de Navarino, aunque es posible que, por su baja frecuencia, no haya sido reportado en algunos casos (Fig. 3d).

También es visible alguna variabilidad regional o algunos sitios que podrían considerarse como de explotación particular de una especie (e.g. RC1 explotación de peces en la estepa o BVS42 explotación de guanacos en P. 

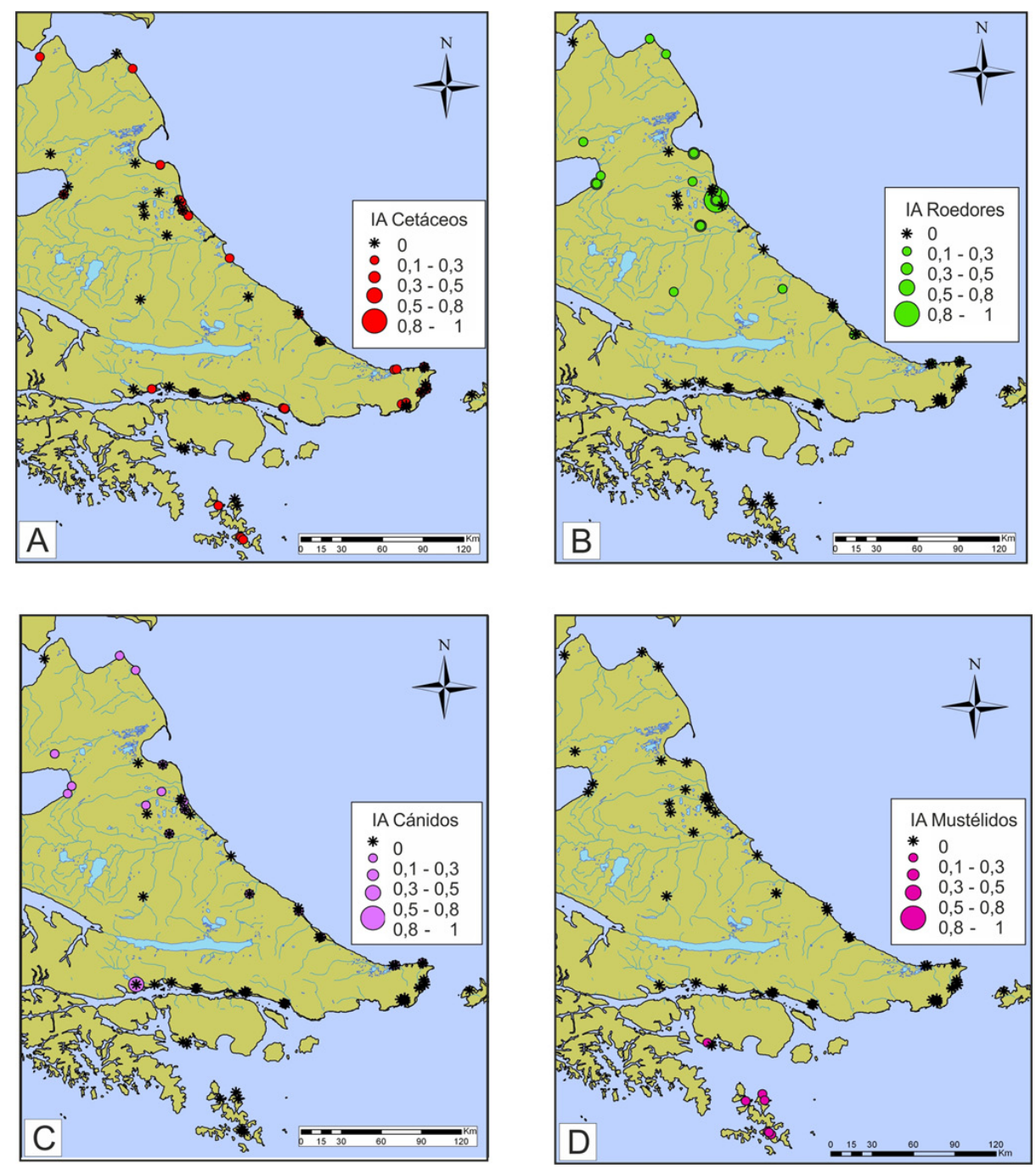

Figura 3: Mapas de distribución de A) índice de abundancia de cetáceos. B) índice de abundancia de roedores. C) índice de abundancia de cánidos. D) índice de abundancia de mustélidos.

Figure 3: Distribution maps of $A$ ) cetaceans index. B) rodents index. C) canids index. D) mustelids index.

fueguino con fines analíticos; dejaremos para un próximo trabajo el análisis de la variabilidad detectada en el plano temporal. Es posible argumentar que esta forma de exponer y explorar los datos nos sirve para filtrar el 'ruido' de fondo de toda la señal zooarqueológica, y ofreciendo una imagen de 'grano grueso' del consumo prehistórico de vertebrados de todo el archipiélago, lo que podríamos caracterizar como la "dieta promedio" de los habitantes del archipiélago fueguino. Este procedimiento conlleva la desventaja de la pérdida de resolución, dado que hay una disminución en la habilidad de detectar las variaciones, pese a lo cual lo consideramos una vía de entrada válida como primera Mitre), en el medio de una región en donde la generalidad es la explotación de otra/s especie/s.

\section{Discusión y conclusiones}

Teniendo en cuenta los potenciales sesgos tafonómicos o metodológicos señalados más arriba, consideramos al NISP como una herramienta útil, por el momento la única disponible, para llevar a cabo un análisis de la escala propuesta en el presente trabajo, ya que presenta más ventajas comparativas (con MNE y MNI) que limitaciones (Grayson 1984, Lyman 2008). Por otra parte, el potencial analítico que brindan los sistemas de información geográfica permite una sistematización de la información y versatilidad para el análisis que resultaron óptimas para los objetivos planteados.

Casi la totalidad del registro arqueofaunístico es un palimpsesto o un conjunto tiempo-promediado, representando múltiples generaciones, múltiples estaciones, múltiples años, y múltiples décadas, incluso centurias o milenios (Lyman 2008). En nuestro caso nos hemos centrado en un solo plano de variabilidad, el espacial, realizando un único promediado de tiempo y exponiendo todos los datos juntos del archipiélago aproximación al problema de la explotación de recursos a una escala supraregional.

Interpretamos estos patrones geográficos de consumo de vertebrados descriptos en el acápite anterior, como el reflejo de tradiciones de caza-recolección regionales especializadas. En la estepa una especialización en la caza y consumo de guanaco (Borrero 1986, Santiago 2010), en península Mitre un mayor ingreso de proteínas de aves y pinnípedos (Vázquez et al. 2011) y en el canal Beagle una tendencia de consumo de pinnípedos por sobre el resto de los taxones representados (Orquera y Piana 1999, Orquera et al. 2008, entre otros). Las islas exteriores presentan un mayor consumo de aves, seguidas de pinnípedos y la incorporación de mustélidos (Legoupil 1993).

Estos modos de subsistencia pueden haber estado influenciados por la estructura ecológica subyacente a cada una de las regiones y por la tecnología que cada una de las sociedades tenía disponible para explotar el medio ambiente. Los cazadores-recolectores tomaron decisiones Acerca de qué recursos explotar, basándose en todo el abanico de opciones aprovechables en un medioambiente dado, como así también teniendo en cuenta las opciones 
tecnológicas y la información de la que disponían.

El patrón, o mejor dicho los patrones, de consumo diferencial de animales detectados, a primera vista bien pueden superponerse con el conocimiento que tenemos de la etnografía de Tierra del Fuego de momentos históricos con las sociedades Selk'nam, Haush, Yámanas y Alakalufes respectivamente. Este patrón también es observable arqueológicamente en los análisis de isótopos estables sobre huesos humanos de toda la región (Guichón et al. 2001, Panarello et al. 2006, Yesner et al. 2003).

Sin embargo, si intentamos desprendernos de la clásica categorización etnográfica encontramos un panorama que bien podría ajustarse a otras posibles "disecciones" territoriales, que no necesariamente responden a la clásica discriminación Selk'nam-Haush o Yámana-Haush. Si bien cabe aclarar que no es nuestra intención discutir la validez de tales categorías, sí queremos enfatizar el grado de variación que encontramos dentro de los territorios asignados tradicionalmente a dichas configuraciones étnicas.

A priori no sorprende la existencia de sitios relativamente especializados en la explotación de guanacos en el norte o centro de la isla, pero sí lo hacen contextos donde la dominancia de este taxón es casi absoluta en otros espacios como bahía Valentín (BVS42, Vázquez et al. 2010) o canal Beagle (Isla el Salmon, Figuerero Torres y Mengoni 1986 o $1^{\text {er }}$ pulso de Shamakush VIII, Piana y Vázquez 2004). Similar razonamiento se podría aplicar a la masiva presencia de peces en sitios del norte y centro de la costa atlántica (Punta Baja 7, Lucero 2009 o Río Chico 1, Santiago 2010).

En el mismo sentido, es destacable la variación expresada dentro de territorios que, a priori y siguiendo la bibliografía etnográfica, mantendrían cierta unicidad desde el punto de vista étnico. Es el caso de la península Mitre en la cual se manifiestan tendencias diferenciadas si comparamos por separado "sub unidades" de esta región, como podrían ser sus litorales norte y sur. Lo mismo sucede en el territorio definido como Yámana, donde la variabilidad en la composición de las arqueofaunas se manifiesta en distintas escalas: regional, local y hasta intrasitio.

La incorporación de componentes marinos a la dieta de los cazadores recolectores fueguinos sea cual fuere su asignación étnica o cronológica, es una constante señalada por muchos autores y constatada desde diversas líneas de evidencia (etnográfica, isotópica y zooarqueológica). El análisis realizado en este trabajo refuerza estas afirmaciones, pero nos interesa destacar el espectro de variación en que los diferentes componentes o categorías de recursos marinos se articulan entre sí y a su vez con los terrestres a lo largo del territorio. Explorar las condiciones que determinaron la generación de contextos taxonómicamente muy diversos o, en el otro extremo, con claras dominancias de una clase de recurso sobre otro, queda fuera de la perspectiva de este trabajo; pero consideramos esta recopilación como un aporte hacia la comprensión y caracterización de la verdadera gama de las adaptaciones fueguinas.

Esta primera imagen nos puede servir para dirigir nuevas preguntas a nivel supraregional. Como por ejemplo: ¿hubo cambios en el consumo de vertebrados en las diferentes regiones del archipiélago? O ¿hubo cambios a nivel particular de una región o microrregión?

Contestando brevemente a la pregunta mencionada, otros autores han detectado que a lo largo del tiempo hubo cambios de consumo en algunas de las regiones de la isla. Solamente para mencionarlos se pudo observar que en el norte de la isla hay un incremento en el consumo de guanacos a través del tiempo (Santiago 2010), en el canal Beagle hay un incremento de la presencia de aves y peces en detrimento de los pinnípedos en tiempos recientes (Zangrando 2009, Tivoli 2010). En otros trabajos exploraremos este plano de variabilidad que por cuestiones de espacio no desarrollamos aquí.

El uso humano de los recursos y del paisaje en el archipiélago fueguino ha sido variado, como lo muestran las arqueofaunas analizadas. Aunque como dijimos, podemos superponer estos datos con los registros etnográficos, reduciendo la variación de lo que observamos a las categorías que conocemos (Borrero 2011), si analizamos los datos haciendo 'zoom' en alguna región en particular observamos mucha mayor variabilidad de lo que esperábamos. Quizás estas nuevas herramientas metodológicas (utilización del GIS para manejo de grandes cantidades de datos) nos ayuden a comprender mejor el complejo consumo prehistórico de vertebrados a lo largo y ancho del archipiélago fueguino.

Ushuaia, 24 de febrero de 2012

\section{Agradecimientos}

Deseamos agradecer las charlas y discusiones con $\mathrm{F}$. Zangrando, M. Salemme, E. Piana. También la ayuda técnica y resolución de algunos problemas con el GIS de A. Raya Rey y P. Torres Carbonell. Los comentarios de A.M. Tívoli y un revisor anónimo que mejoraron el texto original. Todo lo aquí expuesto es nuestra responsabilidad.

\section{Bibliografía}

Barberena, R. 2004. Arqueología e isótopos estables en Tierra del Fuego. Temas de Arqueología Arqueología del norte de la Isla Grande de Tierra del Fuego. Editado por Borrero L. A. y R. Barberena, 135-169. Dunken. Buenos Aires.

Borrero, L. A. 1985. “La Economía Prehistórica de los 
Habitantes del Norte de la Isla Grande de Tierra del Fuego". Fac de Filos y Letras, Universidad de Buenos Aires. UBA. MS. Tesis de Doctorado. 390 + anexos.

Borrero, L. A. 1989. Replanteo de la arqueología patagónica. Interciencia. 14, (3):127-135.

Borrero, L. A. 1990. Fuego Patagonia bone assemblage and the problem of communal guanaco hunting. Hunter of the recent past. Editado por Davis L. B. y B. Reeves, 373-339. Unwin Hyman.

Borrero, L. A. 2003. Taphonomy of the Tres Arroyos 1 Rockshelter, Tierra del Fuego, Chile. Quaternary International. 109, 87-93.

Borrero, L. A. 2005. Taphonomy of Late Pleistocene faunas at Fuego-Patagonia. Journal of South American Earth Sciences. 20: 115-120.

Borrero, L. 2011. Los cazadores orientales de Tierra del Fuego. Los cazadores-recolectores del extremo oriental fueguino Arqueología de Península Mitre e Isla de los Estados Editado por Zangrando A. F., M. Vázquez y A. Tessone, 287-298. Sociedad Argentina de Antropología. Buenos Aires.

Borrero, L. A., M. Casiraghi y H. D. Yacobaccio, 1985. First Guanaco-Processing Site in Southern South America. Current Anthropology. 26, (2):273-276.

Borrero, L. A., A. Martín, V. Horwitz, N. Franco, C. Favier Dubois, F. Borella, F. Carballo Marina, J. B. Belardi, P. Campán, R. A. Guichón, A. S. Muñoz, R. Barberena, F. Savanti y K. Borrazo, 2006. Arqueología de la costa norte de Tierra del Fuego. Arqueología de la costa Patagónica Perspectivas para la conservación. Editado por Caracotche M. y B. Ladrón De Guevara, 251-265. UNPA. Río Gallegos.

Bujalesky, G. G. 2007. Coastal Geomorphology and Evolution of Tierra del Fuego (Southern Argentina). Geologica Acta Vol 4. N 5:337-362.

Calás, E. 2009. “La subsistencia de los cazadoresrecolectores terrestres del Holoceno Medio y Tardío en el norte de Tierra del Fuego". Facultad de Ciencias Sociales Departamento de Antropología. Universidad de Chile. MS. Tesis de Licenciatura. 202.

Calás, E. y M. J. Lucero, 2009. El sitio Marazzi 2: una ocupación costera de cazadores terrestres. Arqueología de Patagonia Una mirada desde el último confín. Editado por Salemme M., F. Santiago, M. Alvarez, E. L. Piana, M. Vázquez y M. E. Mansur, II. 913-932. Editorial Utopías. Ushuaia.

Camarós, E., V. Parmigiani y E. Verdún, 2009. Espacio ritual, espacio domestico: diferencias en uso del recurso faunístico en la sociedad Selk'nam. Arqueología de la Patagonia Una mirada desde el último confín. Editado por Salemme M., F. Santiago, M. Alvarez, E. Piana, M. Vázquez y E. Mansur, II. 667-675. Editorial Utopías. Ushuaia.

Chapman, A. 1987. La isla de los estados en la prehistoria. Primeros datos arqueológicos. Eudeba. Buenos Aires.

Estevez, J., E. Piana, A. Schiavini y N. Juan-Muns, 2001. Archaeological analysis of shell middens in the Beagle Channel, Tierra del Fuego Island. International Journal of Osteoarchaeology. 11, (1-2):24-33.

Figuerero Torres, M. J. 1988. Nuevos fechados para las ocupaciones de la Isla El Salmón, Parque Nacional Tierra del Fuego. 113.

Figuerero Torres, M. J. y L. Mengoni Goñalons. 1986. Excavaciones arqueológicas en la Isla El Salmon. Parque Nacional Tierra del Fuego. PREP Informes de Investigación. Buenos Aires. 94.

Grayson, D. 1984. Cuantitative Zooarchaeology. Academic Press, New York.

Guichón, R. A., L. A. Borrero, A. Prieto, P. Cárdenas y R. Tykot, 2001. Nuevas determinaciones de isótopos estables para Tierra del Fuego. Revista Argentina de Antropología Biológica. 3, (1):113-126.

Heusser, C. J. 2003. Ice Age Southern Andes. A chronicle of paleoecological events. Elsevier

Horwitz, V. 1995. Ocupaciones prehistóricas en el sur de Bahía San Sebastián (Tierra del Fuego, Argentina). Arqueología. 5, 105-136.

Horwitz, V. 1996-1998. Espíritu Santo: primeros trabajos de campo en el extremo norte de la costa atlántica fueguina. Palimpsesto. 5, 151-159.

Horwitz, V. 2004. Arqueología de la costa atlantica septentrional de Tierra del Fuego. Temas de Arqueología Arqueología del norte de la Isla Grande de Tierra del Fuego. Editado por Borrero L. A. y R. Barberena, 29-54. Dunken. Buenos Aires.

Horwitz, V., L. A. Borrero y M. Casiraghi. 1993/1994. Estudios arqueológicos en San Julio 2 (Tierra del Fuego). Relaciones de la Sociedad Argentina de Antropología. XIX, 391-416.

Laming Emperaire, A., D. Lavallée y R. Humbert. 1972. Le site de Marazzi en Terre de Feu. Objets et Mondes. $12,225-244$. 
Lanata, J. L. 1995. Paisajes Arqueológicos y Propiedades del Registro en el Sudeste Fueguino. Departamento de Antropología. UBA. MS. Doctoral. 256.

Lanata, J. L. 1996. The "Haush" puzzle: piecing together subsistence and settlement at the Fueguian southeast. Revista do Museu de Arqueologia e Etnología. 6, 11-32.

Lanata, J. L. y A. Winograd. 1988. Gritos y susurros: aborígenes y lobos marinos en el litoral de la Tierra del Fuego. Fondo de Promoción de la Cultura. Bogotá. 227-246.

Legoupil, D. 1993. El Archipielago del Cabo de Hornos y la Costa Sur del la Isla Navarino: Poblamiento y Modelos económicos. Anales del Instituto de la Patagonia (serie Ciencias Humanas). 22, 101-121.

Lyman, R. L. 2003. The influence of time averaging and space averaging on the application of foraging theory in zooarchaeology. Journal of Archaeological Science. 30, 595-610.

Lyman, R. L. 2008. Quantitative Paleozoology. Cambridge University Press. New York.

Mansur, M. E., D. Martinioni y A. Lasa. 2000. La gestión de los recursos líticos en el sitio Marina I (zona central de Tierra del Fuego, Argentina). Desde el País de los Gigantes Perspectivas Arqueológicas en Patagonia. Editado por Belardi J. B., F. Carballo Marina y S. Espinosa, I. 57-72. UNPA. Río Gallegos.

Massone, M. F. 2004. Los cazadores despues del hielo. Colección de Antropologia VII. Centro de Investigaciones Diego barros Arana, DIBAM, Santiago.

Massone, M. F. 2010. Los cazadores del viento. Selk'nam. TurismoChile. Santiago.

Massone, M., F. Morello, A. Prieto, M. San Román, A. Martín y P. Cárdenas. 2003. Sitios arqueológicos, restos de cetáceos y territorios locales Selk'nam en Bahía Inutil, Tierra del Fuego. Magallania. 31, 45-59.

Miotti, L. y M. Salemme. 1999. Biodiversity, taxonomic richness and specialists-generalists during Late Pleistocene/ Early Holocene times in Pampa and Patagonia (Argentina, Southern South America) Quaternary International. 53/54, 53-68.

Morello, F., L. Contreras y M. San Román. 1999. La localidad de Marazzi y el sitio arqueológico Marazzi I, una re-evaluación. Anales del Instituto de la Patagonia (serie Ciencias Humanas). 27, 183-197.

Muñoz, A. S. y J. B. Belardi. 2011. Nueva información sobre viejos datos: arqueología del norte de Península
Mitre. Los cazadores-recolectores del extremo oriental fueguino Arqueología de Península Mitre e Isla de los Estados Editado por Zangrando A. F., M. Vázquez y A. Tessone, 171-202. Sociedad Argentina de Antropología. Buenos Aires.

Muñoz, A.S. 2002. "La explotación de Mamíferos por cazadores-recolectores terrestres de Tierra del Fuego". Fac. de Filosofía y Letras. UBA. MS. Tesis de Doctorado.

Muñoz, S. A. 2004. Mammal exploitation in the insular environments of southern South America. Zooarchaeology of South America. Editado por Mengoni Goñalons L., 123-138. BAR International Series 1298. Archaeopress

Orquera, L. A. y E. Piana. 1988. Human littoral adaptation in the Beagle Channel region: maximum possible age. Quaternary of South America and Antarctic Peninsula. 5, 133- 162

Orquera, L. A. y E. L. Piana. 1996. El sitio Shamakush I. Relaciones de la Sociedad Argentina de Antropología. XXI, 215-265.

Orquera, L. A. y E. L. Piana. 1999. Arqueología de la región del canal Beagle (Tierra del Fuego, República Argentina). Sociedad Argentina de Antropología. Buenos Aires.

Orquera, L. A. y E. L. Piana. 2009. Sea Nomads of the Beagle Channel in Southernmost South America: Over Six Thousand Years of Coastal Adaptation and Stability. The Journal of Island and Coastal Archaeology. 4, (1):61-81.

Orquera, L.A., E.L. Piana, M.R. Álvarez, D. Fiore, M.M. Vázquez, A.F.J. Zangrando, A. Tessone y A.M. Tivoli. 2006. El Proyecto Arqueológico Canal Beagle. Arqueología de la Costa Patagónica. Perspectivas para la Conservación: 266-288. Editado por I. Cruz y S. Caracotche, Universidad Nacional de la Patagonia Austral - Subsecretaría de Cultura Provincia de Santa Cruz, Río Gallegos.

Panarello, H., A. F. Zangrando, A. Tessone, L. Kozameh y N. Testa. 2006. Análisis comparativo de paleodietas humanas entre la región del Canal de Beagle y Península Mitre: Perspectivas desde los isótopos estables. Magallania. $34,(2): 37-46$

Piana, E. y G. Canale. 1993-94. Tunel II Un yacimiento de la fase reciente del Canal Beagle. Relaciones de la Sociedad Argentina de Antropología. XIX, 363-389.

Piana, E. y M. Vázquez. 2004. Shamakush VIII. Puntualizaciones sobre el uso de recursos y la gestión del asentamiento en el Canal Beagle. XV Congreso Nacional de Arqueología Argentina. Editado por Tamagnini M. y O. Mendonca, UNRC. Río Cuarto. 
Piana, E., M. Vázquez y N. Rua. 2004. Mischiuen I primeros resultados de una excavación de rescate en la costa norte del Canal Beagle. Contra viento y marea Arqueología de Patagonia. Editado por Civalero M. T., P. Fernández y A. G. Guráieb, 815-832. Ediciones Instituto Nacional de Antropología y Pensamiento Latinoamericano. Buenos Aires.

Piana, E., M. Vázquez, M. Álvarez y N. Rua. 2007. El sitio Ajej I: Excavación de rescate en la costa del Canal Beagle. Arqueología Argentina en los inicios de un nuevo siglo. Editado por Oliva A., N. De Grandis y J. Rodríguez, 1. 345-356. UNR Facultad de Humanidades y Artes. Escuela de Antropología. Rosario.

Piana E. L. , M. Vazquez y A. Tivoli. 2007. Dieta y algo mas. Animales pequeños y variabilidad del comportamiento humano en el canal Beagle. Arqueología de FuegoPatagonia, levantando piedras, desenterrando huesos... y develando arcanos. Ediciones CECUA. Punta Arenas. Chile.

Ponce, F., A. M. Borromei y J. Rabassa. 2011. Evolución del paisaje y la vegetación durante el Cenozoico tardío en el extremo sureste del archipiélago fueguino y canal Beagle. Los cazadores-recolectores del extremo oriental fueguino Arqueología de Península Mitre e Isla de los Estados. Editado por Zangrando A. F., M. Vázquez y A. Tessone, 31-64. Sociedad Argentina de Antropología. Buenos Aires.

Prieto, A., E. Calás, F. Morello y J. Torres. 2007. El sitio arqueológico Myren 2, Tierra del Fuego, Chile. Magallania. 35, (2):89-103.

Salemme, M., G. Bujalesky y F. Santiago. 2007. La Arcillosa 2: la ocupación humana durante el Holoceno medio en el Río Chico, Tierra del Fuego, Argentina. Arqueología de Fuego-Patagonia Levantando piedras, desenterrando huesos y develando arcanos. Editado por Morello F., M. Martinic, A. Prieto y G. Bahamonde, 723-736. Ediciones CEQUA. Punta Arenas (Chile).

Santiago, F. 2010. "La ocupación humana del norte de Tierra del Fuego durante el Holoceno medio y Tardío. Su vinculación con el paisaje". Facultad de Ciencias Sociales. UNICEN. MS. Tesis de Doctorado. 245.

Santiago, F. y M. Salemme. 2010. Sondeos, recolecciones y excavaciones arqueológicas en Las Vueltas 1 (Tierra del Fuego, Argentina). Arqueología Argentina en el Bicentenario de la Revolución de Mayo. Editado por Bárcena R. y H. Chiavazza, V. 1975-1980. Facultad de
Filosofia y Letras UNCuyo. Mendoza.

Santiago, F., M. Salemme, J.A. Suby y R. Guichón. 2011. Restos óseos humanos en el norte de Tierra del Fuego. Aspectos contextuales, dietarios y paleopatológicos. Intersecciones en Antropología. 12, 156-170.

Schiavini, A. 1993. Los lobos marinos como recurso para cazadores-recolectores marinos: El caso de Tierra del Fuego. Latin American Antiquity 4: 346-366.

Tivoli, A. M. 2010. Exploitation of bird resources among prehistoric sea-nomad societies of the Beagle Channel region, southern South America. Before Farming. 2, (3):1-12.

Vázquez, M., A. F. Zangrando, A. Tessone, A. Ceraso y L. Sosa. 2007. Arqueología de Bahía Valentín (Península Mitre, Tierra del Fuego): nuevos resultados y perspectivas. Arqueología de Fuego-Patagonia Levantando piedras, desenterrando huesos develando arcanos. Editado por Morello F., A. Martinic, A. Prieto y G. Bahamonde, 755766. Ediciones CEQUA. Punta Arenas.

Vázquez, M., A. F. Zangrando, A. Tessone y A. Ceraso. 2011. Arqueologia de la costa meridional de Peninsula Mitre. Los cazadores-recolectores del extremo oriental fueguino Arqueología de Península Mitre e Isla de los Estados. Editado por Zangrando A. F., M. Vázquez y A. Tessone, 203-230. Sociedad Argentina de Antropología. Buenos Aires.

Yesner, D., M. J. Figuerero Torres, R. A. Guichón y L. A. Borrero. 2003. Stable isotope analysis of human bone and ethnohistoric subsistence patterns in Tierra del Fuego. Journal of Anthropological Archaeology. 22, 279-291.

Zangrando, A. F. 2009. Historia evolutiva y subsistencia de cazadores-recolectores marítimos de Tierra del Fuego. Sociedad Argentina de Antropología. Buenos Aires.

Zangrando, A. F. 2010. Coastal Archaeology and HunterGatherers in Southeastern Tierra del Fuego. Journal of Island and Coastal Archaeology. 5 (2):288-291.

Zangrando, A. F., D. Alumni, M. P. Martinoli, A. M. Tivoli y E. Piana. 2010. Arqueología de la región de Moat (Tierra del Fuego, Argentina): Estudios preliminares en la localidad arqueológica Heshkaia. Arqueología Argentina en el Bicentenario de la Revolución de Mayo. Editado por Bárcena R. y H. Chiavazza, V. 2005-2010. UNCu. Mendoza. 